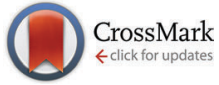

Cite this: Chem. Commun., 2015, 51,3069

Received 11th November 2014, Accepted 6th January 2015

DOI: $10.1039 / c 4 c c 09008 j$

www.rsc.org/chemcomm

\section{Single molecule level plasmonic catalysis - a dilution study of $p$-nitrothiophenol on gold dimers $\dagger$}

\author{
Zhenglong Zhang, ${ }^{a}$ Tanja Deckert-Gaudig, ${ }^{a}$ Pushkar Singh ${ }^{a}$ and Volker Deckert*ab
}

\begin{abstract}
Surface plasmons on isolated gold dimers can initiate intermolecular reactions of adsorbed $p$-nitrothiophenol. At the single molecule level when dimerization is not possible an intramolecular reaction can be observed. Experimental evidence indicates that plasmon-induced hot electrons provide the required activation energy.
\end{abstract}

In the presence of surface plasmons molecular chemical reactions can be investigated at the nanoscale, as it has been demonstrated for a variety of catalyzed systems. ${ }^{1-19}$ Recently, plasmon catalyzed dimerization of $p$-nitrothiophenol ( $p$ NTP) to 4,4-dimercaptoazobenzene (DMAB) has been realized using surface-enhanced Raman spectroscopy (SERS) and tip-enhanced Raman spectroscopy (TERS). ${ }^{2-6}$ For any dimerization the distance between the reactants is decisive. If the distance between $p$ NTP molecules gets too large a dimerization reaction cannot occur. In this case the question arises, whether surface plasmons can initiate a further intramolecular reaction like dissociation or rearrangement. To address this issue, a detection sensitivity of signals from a few or ultimately even a single educt molecule is required. In our experiments we employed SERS, which is widely used for noninvasive detection, biological and chemical sensing..$^{20-25}$ In SERS laser irradiation of silver or gold nanoparticles induces localized surface plasmon resonances at the nanostructured surface generating enhanced electromagnetic fields. ${ }^{25}$ If molecules are brought in closest vicinity to such nanoparticles (e.g. by adsorption) a large enhancement of the Raman scattering signal can be observed. Using the junctions or gaps of nanoparticles, so-called "hot spots", very high enhancement factors $\left(\sim 10^{8}\right)$ can be achieved, providing sufficient signal enhancement for single molecule detection. Therefore such junctions are ideally suited for the intended reactivity detection. ${ }^{23-27}$

\footnotetext{
${ }^{a}$ Leibniz Institute of Photonic Technology, Albert-Einstein-Str. 9, 07745 Jena, Germany. E-mail: volker.deckert@ipht-jena.de

${ }^{b}$ Institute of Physical Chemistry and Abbe Center of Photonics, Friedrich-Schiller University Jena, Helmholtzweg 4, 07743 Jena, Germany $\dagger$ Electronic supplementary information (ESI) available: Experimental details and Fig. S1-S3. See DOI: 10.1039/c4cc09008j
}

In previous studies, it was found that the plasmon catalyzed intermolecular reaction of $p$ NTP (adsorbed on gold or silver nanoparticles) to DMAB can be controlled by laser intensity. ${ }^{3,4}$ A higher laser intensity generates a stronger plasmon resonance with higher kinetic energy yielding an increased reaction rate. If the concentration, however, is low enough to prevent clustering and self-assembly, the distance of $p$ NTP molecules adsorbed on the nanoparticles should become too large for an intermolecular reaction and no reaction should occur even at high laser powers. Then, any detected SERS signal can be related to single or only a few isolated and non-interacting separated molecules.

In this communication, single gold nanoparticle dimers were employed for SERS detection of $p$ NTP molecules. In the presented experiments a so far unknown behavior of $p$ NTP could be monitored, which strongly differs from previous reports as separated $p$ NTP molecules exclusively reacted to thiophenol (TP), and is a new finding in surface catalyzed experiments. In addition, a step-like signal intensity change during the process strongly indicates that the dissociation reaction of $p$ NTP to TP occurs at or close to a single molecule level.

As shown in Fig. 1, SERS active gold nanoparticle dimers (GDs) were prepared using a wet chemistry method. ${ }^{28}$ Further experimental details can be found in the ESI. $\dagger$ In order to achieve SERS of separated molecules, three different concentrations of aqueous $p$ NTP solutions $\left(5 \times 10^{-7}, 10^{-8}\right.$ and $\left.10^{-9} \mathrm{M}\right)$ were mixed with
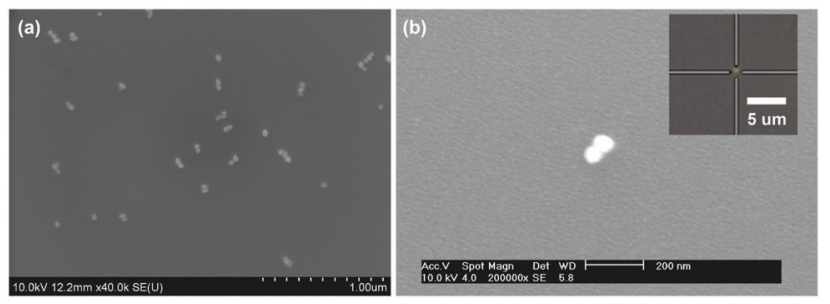

Fig. 1 (a) SEM image of gold nanoparticle dimers spin-coated onto a silicon wafer, (b) a single gold nanoparticle dimer on glass as used in the SERS measurement (inset: optical image of the selected nanoparticle dimer, which is placed in the center of the laser focus). 

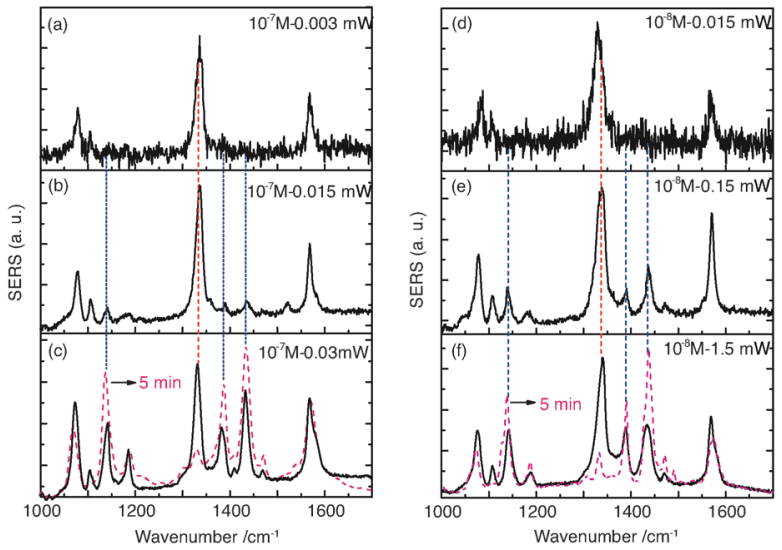

Fig. 2 (a)-(c) SERS spectra of $10^{-7} \mathrm{M}$ pNTP excited at 3, 15 and $30 \mu \mathrm{W}$ laser power, respectively. (d)-(f) SERS spectra of $10^{-8} \mathrm{M}$ pNTP excited at $0.015,0.15$ and $1.5 \mathrm{~mW}$ laser power, respectively. The dotted red curves in (c) and (f) refer to spectra after continuous irradiation for 5 minutes.

a gold nanoparticle dimer colloidal solution. The dimerization of $p$ NTP to DMAB is characterized by the disappearance of the Raman band at $1332 \mathrm{~cm}^{-1}\left(\nu_{\mathrm{NO}_{2}}\right)$, and the appearance of new Raman bands around $1140\left(\beta_{\mathrm{C}-\mathrm{H}}\right), 1387\left(\nu_{\mathrm{NN}}+\nu_{\mathrm{CC}}+\nu_{\mathrm{C}-\mathrm{N}}\right)$ and $1432\left(\nu_{\mathrm{NN}}+\nu_{\mathrm{CC}}+\beta_{\mathrm{C}-\mathrm{H}}\right) \mathrm{cm}^{-1}$, which are related to the $-\mathrm{N}=\mathrm{N}-$ unit of DMAB. ${ }^{2}$

First, SERS experiments of the $10^{-7} \mathrm{M} p \mathrm{NTP}-\mathrm{GD}$ mixture were made with increasing laser intensity. Starting with $3 \mu \mathrm{W}$ (see Fig. 2(a)) only the three main peaks of $p$ NTP were detected in the SERS spectrum. Increasing the laser power to $15 \mu \mathrm{W}$ and $30 \mu \mathrm{W}$, respectively, dimerization of $p$ NTP to DMAB was verified by the afore-mentioned bands at 1140, 1387 and $1432 \mathrm{~cm}^{-1}$ (Fig. 2(b)). Fig. 2(b) and (c) indicate also that an increased laser power yields a higher reaction rate. After continuous irradiation for 5 min almost all $p$ NTP molecules in the laser spot have reacted to DMAB (see dotted red curve in Fig. 2(c)).

In the next experiment the $10^{-8} \mathrm{M} p \mathrm{NTP}-\mathrm{GD}$ mixture were investigated, all other experimental parameters except the laser power as stated below remained unchanged. The corresponding SERS spectra are shown in Fig. 2(d)-(f). Similar to the previous experiment, a dimerization of $p$ NTP to DMAB occurred, however, higher laser intensities $(150 \mu \mathrm{W})$ were needed to initiate the reaction. The results of both experiments demonstrate that with $10^{-7} \mathrm{M}$ and $10^{-8} \mathrm{M} p \mathrm{NTP}$ solutions the gold surface is still densely covered with $p$ NTP molecules enabling a dimerization.

For the final experiment a $10^{-9} \mathrm{M} p$ NTP solution was used. The resulting SERS spectra are shown in Fig. 3(a)-(c). During these measurements no dimerization of $p$ NTP to DMAB was observed. Even at highest laser power $(3 \mathrm{~mW})$ for one hour (see Fig. 3(d)-(h) and for the full trace ESI, † Fig. S1) no DMAB signals could be detected. It was concluded that the distance between $p$ NTP molecules in the plasmonic hot-spot of the GD was indeed too large to allow intermolecular reactions under these conditions. That the reaction of $p$ NTP to DMAB could not be initiated even after 1 hour of continuous irradiation, indicates that SERS signals from only a few separated molecules in the hot-spot were acquired. This finding is in accordance with the estimated
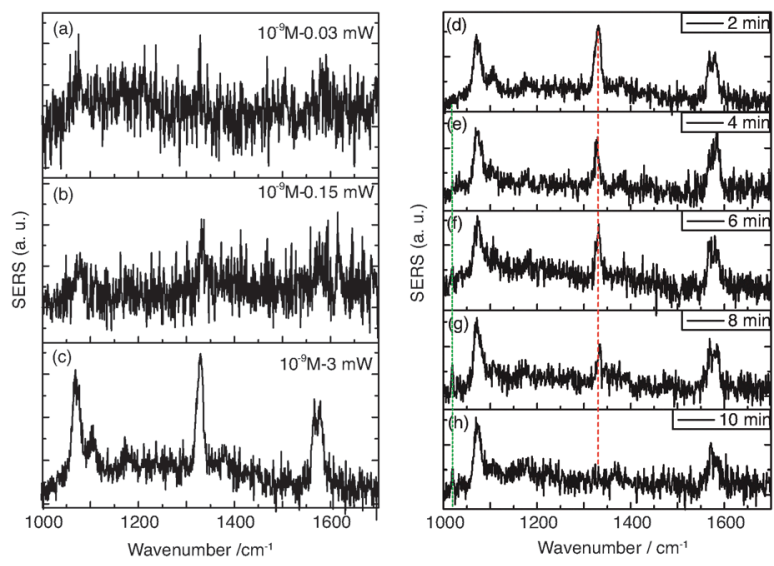

Fig. 3 (a)-(c) Laser intensity dependent SERS spectra excited at 0.03, 0.15 and $3 \mathrm{~mW}$ laser power, respectively. (d) -(h) Time dependent SERS spectra at $3 \mathrm{~mW}$ laser at the sample.

molecular coverage $n$ of $p$ NTP on a single gold dimer that was estimated as follows:

$$
n=\frac{n_{\mathrm{M}}}{n_{\mathrm{GD}}}=\frac{c_{\mathrm{M}}}{c_{\mathrm{GD}}}=c_{\mathrm{M}} / \frac{m_{\mathrm{Au}}}{m_{\mathrm{GD}} \cdot N_{\mathrm{A}} \cdot V}
$$

where $c_{\mathrm{M}}$ and $c_{\mathrm{GD}}$ are the molarity of $p$ NTP and GD nanoparticles, $m_{\mathrm{Au}}$ and $m_{\mathrm{GD}}$ refer to the mass of Au and one single GD nanoparticle, respectively. $N_{\mathrm{A}}$ is Avogadro's constant; $V$ is the volume of GDs solution in the preparation process. According to the SEM images the diameter of gold nanoparticles is about $40 \mathrm{~nm}$, and the molarity of the GDs solution is about $3.7 \times 10^{-10} \mathrm{M}$. Consequently, there are approximately 1000, 100 and 10 molecules absorbed on a single nanoparticle dimer for the respective $10^{-7}, 10^{-8}$ and $10^{-9} \mathrm{M}$ solutions. Since not all molecules can be absorbed on GDs in the experiment, the actual number of molecules on average will be even lower in the experiments. From the absence of dimerization in the low concentration case we can also conclude that the molecules do not cluster and are statistically absorbed on the GD surface. The average distance $d$ between two molecules can be estimated as

$$
d=R \sqrt{8 \pi / n} .
$$

$R$ is the radius of the gold nanoparticle and $n$ is the number of molecules absorbed on single nanoparticle dimer, which can be calculated by eqn (1). The calculated distances between two molecules of $10^{-7}$ and $10^{-8} \mathrm{M}$ are $\sim 3 \mathrm{~nm}$ and $10 \mathrm{~nm}$, respectively, which is either close enough for two $p$ NTP molecules to dimerize to DMAB or, more likely, at such distances collisions happen often enough for a dimerization. But for the $10^{-9} \mathrm{M}$ solution, the average distance between two molecules is $\sim 32 \mathrm{~nm}$, which is obviously preventing the dimerization of two $p$ NTP molecules. Thus, SERS spectra of separated $p$ NTP and the absence of DMAB molecules at the lowest concentration used here can be explained.

Probably the most striking observation in the SERS experiments of $p$ NTP at $10^{-9} \mathrm{M}$ was an intensity decrease of the dominating band at $1332 \mathrm{~cm}^{-1}\left(\nu_{\mathrm{NO}_{2}}\right)$ over time. This peak disappeared after $10 \mathrm{~min}$, simultaneously a new peak at $1017 \mathrm{~cm}^{-1}$ was detected after $5 \mathrm{~min}$, as shown in Fig. 3(d)-(h). Since the $\mathrm{NO}_{2}$ mode of $p$ NTP disappeared, we hypothesize that the nitro group was cleaved from the benzene 


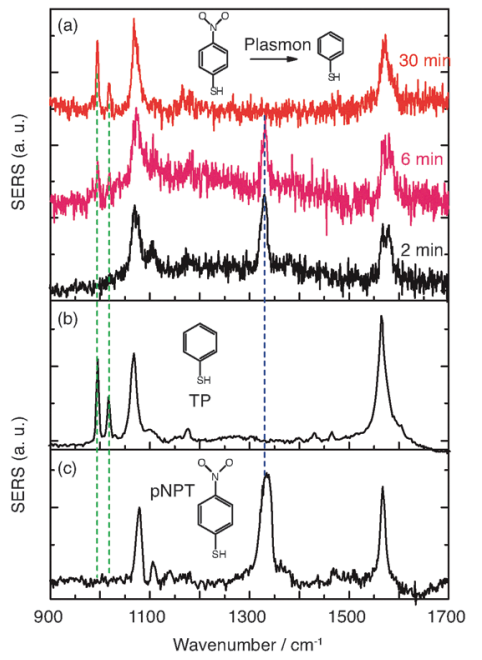

Fig. 4 (a) Time dependent SERS spectra of reacting pNTP $\left(c=10^{-9} \mathrm{M}\right)$ at $3 \mathrm{~mW}$ laser at 2, 6 and $30 \mathrm{~min}$. (b) and (c) normal SERS spectra of TP and pNTP, respectively.

ring in the hot-spot of the gold dimer resulting in the formation of thiophenol (TP). Comparing SERS spectra of neat TP (Fig. 4(b)) with " $p$ NTP on GDs" spectra from late stages (see Fig. $4(\mathrm{a})$ and also Fig. $3(\mathrm{~h}))$ it is obvious that the new bands at $995\left(\beta_{\mathrm{CCC}}\right)$ and $1017\left(\beta_{\mathrm{CH}}\right) \mathrm{cm}^{-1}$ as well as the bands at 420 and $685 \mathrm{~cm}^{-1}$ in the low wavenumber region match those of TP strikingly well (see also ESI, $\dagger$ Fig. S3). The necessary hydrogen atom for the formation of TP was most probably abstracted from the surrounding water layer that was ubiquitously present since $p$ NTP was adsorbed from aqueous solution and the experiment was performed under ambient conditions.

The experimental data demonstrate that a dissociation of $p$ NTP to TP proceeds under our special SERS conditions at a concentration of $10^{-9} \mathrm{M}$ (corresponding to a surface coverage of $\sim 10 p$ NTP molecules per dimer). These results clearly differ from the dimerization reaction of $p$ NTP to DMAB. The light induced dissociation of nitro substituted aromatic molecules is not new but usually requires drastic conditions. For example, the $-\mathrm{NO}_{2}$ group in nitrobenzene and nitrophenol can be cleaved using UV excitation..$^{29-31}$ The required dissociation energy of the $\mathrm{C}-\mathrm{N}$ bond is $\sim 3.03 \mathrm{eV},{ }^{29}$ which is higher than the energy provided by the incident photon of $632.8 \mathrm{~nm}(1.96 \mathrm{eV})$. The power dependent experiments in Fig. 3 show no nonlinear increase of the products, so nonlinear optical effects including two/multiphoton absorption can be ruled out. Hence, the activation energy is too large to be simply overcome by the excitation laser. However, localized surface plasmons excited on silver or gold nanoparticle surfaces can decay non-radiatively into hot electrons with an energy between the vacuum energy and Fermi level $(\sim-5 \mathrm{eV}) \cdot{ }^{32-34}$ Hot electrons can scatter into an excited state of the absorbed molecules, triggering a chemical reaction by reducing the activation energy. ${ }^{34}$ Applied to the present reaction our hypothesis is that plasmon-induced hot electrons initiate $-\mathrm{NO}_{2}$ cleavage from the phenyl ring. A scheme of the proposed mechanism is shown in Fig. 5. Initially, hot electrons generated from plasmon decay on the nanoparticle surface, soon lose coherence and form a

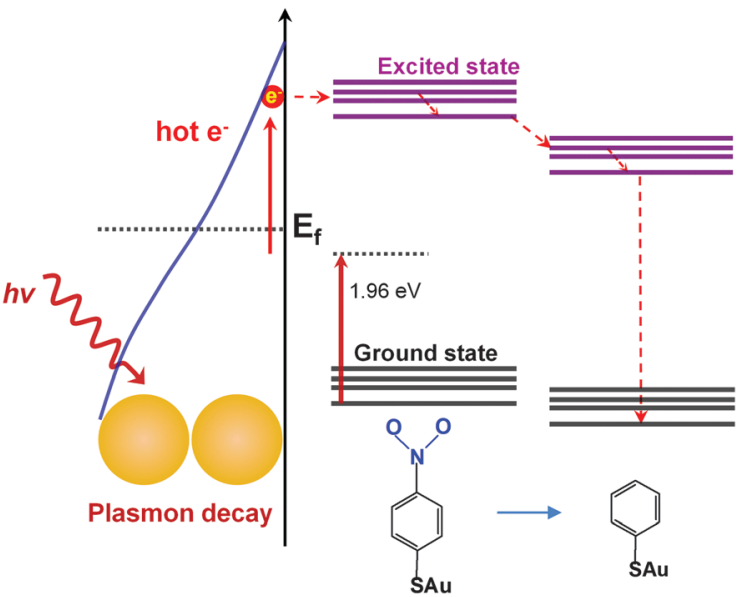

Fig. 5 Proposed mechanism of plasmon (hot electron) catalyzed dissociation of $p$ NTP to TP.

nonequilibrium Fermi-Dirac type distribution. ${ }^{8}$ The hot electrons in the high energy level have sufficient energy to transfer to the excited state of $p$ NTP molecule, creating a transient negative ion of $p$ NTP. This negative ion "travels" to the excited state of TP and transfers the electron back to the GD surface, where it returns to the ground state of TP and dissociates.

It should be noted that the simultaneous detection of $p$ NTP and TP in Fig. 4(a) - spectrum at 6 min - actually points to the presence of at least two molecules in the hot spot. Although the experiment was performed at a very low concentration it cannot be regarded as "single molecule" SERS in the classical sense. According to the time dependent SERS spectra, the peak intensity change $v s$. time is shown for the $p$ NTP band at $1332 \mathrm{~cm}^{-1}$, and the TP band at $1017 \mathrm{~cm}^{-1}$ in Fig. 6, respectively. It was found that three clear steps can be discerned: first, some molecules of $p$ NTP were detected in the first $5 \mathrm{~min}$ of the measurement; after $5 \mathrm{~min}$, half of the $p$ NTP molecules reacted to TP; the remaining $p$ NTP molecules reacted after $8 \mathrm{~min}$. After that no significant change occurred in the remaining experiment (60 min). Consequently, it can be assumed that two distant, non-interacting molecules were detected in the hotspot of a single GD because both $p$ NTP and TP were detected between 5-8 min. Apparently, the two molecules were too far apart from each other for an intermolecular reaction, and instead an intramolecular reaction was initiated. Because of the step-like reaction process, the other possibilities of having $4-2-0,6-3-0$, or 8-4-0 molecules synchronously reacting seem very unlikely. The involvement of even more molecules can be ruled out, as at 10 times higher concentration dimerization already starts.

In summary, we present the detection of a new plasmon catalyzed reaction of $p$ NTP near the single molecule level. A dissociation of $p$ NTP to TP was detected at low concentration where the distance between $p$ NTP molecules adsorbed on single isolated gold dimer became too large for a dimerization reaction. In combination with the results with higher concentrations this further confirms a dimerization reaction of $p$ NTP to DMAB. In accordance with previous reports we assume that plasmon-induced hot electrons provide the activation energy necessary for such a dissociation of $p$ NTP. The tracked step-like 


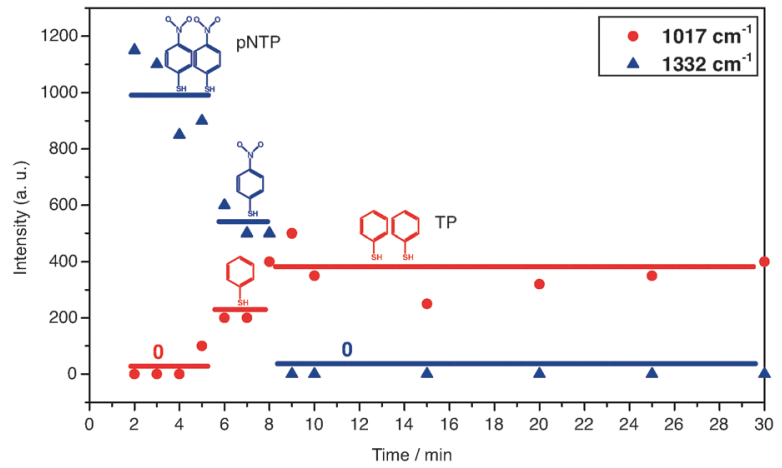

Fig. 6 Step-like process of the dissociation of pNTP to TP.

process of the dissociation of $p$ NTP to TP strongly indicates a single molecule behavior. The results demonstrate that irradiation of an adsorbate in a gold nanoparticle gap can decrease the activation energy barrier such, that a reaction usually requiring UV light can be triggered in the visible.

We gratefully acknowledge support from the Deutsche Forschungsgemeinschaft (DEP4TERS, FR 1348/19-1) and the Thüringer Aufbau Bank (No. 2011SE9048). Z. Zhang acknowledges financial support from the Alexander von Humboldt foundation.

\section{Notes and references}

1 P. Christopher, H. L. Xin and S. Linic, Nat. Chem., 2011, 3, 467-472.

2 B. Dong, Y. R. Fang, X. W. Chen, H. X. Xu and M. T. Sun, Langmuir, 2011, 27, 10677-10682.

3 E. M. van Schrojenstein Lantman, T. Deckert-Gaudig, A. J. G. Mank, V. Deckert and B. M. Weckhuysen, Nat. Nanotechnol., 2012, 7, 583-586.

4 M. T. Sun, Z. L. Zhang, H. R. Zheng and H. X. Xu, Sci. Rep., 2012, 2, 647.

5 V. Joseph, C. Engelbrekt, J. D. Zhang, U. Gernert, J. Ulstrup and J. Kneipp, Angew. Chem., Int. Ed., 2012, 51, 7592-7596.

6 M. T. Sun and H. X. Xu, Small, 2012, 8, 2777-2786.

7 J. Lee, S. Mubeen, X. L. Ji, G. D. Stucky and M. Moskovits, Nano Lett., 2012, 12, 5014-5019.

8 S. Mukherjee, F. Libisch, N. Large, O. Neumann, L. V. Brown, J. Cheng, J. B. Lassiter, E. A. Carter, P. Nordlander and N. J. Halas, Nano Lett., 2013, 13, 240-247.
9 W. Xie, B. Walkenfort and S. Schlucker, J. Am. Chem. Soc., 2013, 135, 1657-1660.

10 M. T. Sun, Z. L. Zhang, Z. H. Kim, H. R. Zheng and H. X. Xu, Chem. - Eur. J., 2013, 19, 14958-14962.

11 K. Ueno and H. Misawa, J. Photochem. Photobiol., C, 2013, 15, 31-52.

12 Z. L. Zhang, M. T. Sun, P. P. Ruan, H. R. Zheng and H. X. Xu, Nanoscale, 2013, 5, 4151-4155.

13 S. Mukherjee, L. A. Zhou, A. M. Goodman, N. Large, C. Ayala-Orozco, Y. Zhang, P. Nordlander and N. J. Halas, J. Am. Chem. Soc., 2014, 136, 64-67.

14 E. M. v. S. Lantman, O. L. J. Gijzeman, A. J. G. Mank and B. M. Weckhuysen, ChemCatChem, 2014, 6, 3342-3346.

15 Z. L. Zhang, S. X. Sheng, H. R. Zheng, H. X. Xu and M. T. Sun, Nanoscale, 2014, 6, 4903-4908.

16 P. Singh and V. Deckert, Chem. Commun., 2014, 50, 11204-11207.

17 C. L. Wang and D. Astruc, Chem. Soc. Rev., 2014, 43, 7188-7216.

18 M. J. Kale, T. Avanesian and P. Christopher, ACS Catal., 2014, 4, 116-128.

19 X. J. Lang, X. D. Chen and J. C. Zhao, Chem. Soc. Rev., 2014, 43, 473-486.

20 D. L. Jeanmaire and R. P. Vanduyne, J. Electroanal. Chem., 1977, 84, 1-20.

21 P. L. Stiles, J. A. Dieringer, N. C. Shah and R. R. Van Duyne, Annu. Rev. Anal. Chem., 2008, 1, 601-626.

22 L. Li, T. Hutter, A. S. Finnemore, F. M. Huang, J. J. Baumberg, S. R. Elliott, U. Steiner and S. Mahajan, Nano Lett., 2012, 12, 4242-4246.

23 S. M. Nie and S. R. Emery, Science, 1997, 275, 1102-1106.

24 K. Kneipp, Y. Wang, H. Kneipp, L. T. Perelman, I. Itzkan, R. Dasari and M. S. Feld, Phys. Rev. Lett., 1997, 78, 1667-1670.

25 H. X. Xu, J. Aizpurua, M. Kall and P. Apell, Phys. Rev. E: Stat. Phys., Plasmas, Fluids, Relat. Interdiscip. Top., 2000, 62, 4318-4324.

26 T. Deckert-Gaudig, E. Kammer and V. Deckert, J. Biophotonics, 2012, 5, 215-219.

27 R. Zhang, Y. Zhang, Z. C. Dong, S. Jiang, C. Zhang, L. G. Chen, L. Zhang, Y. Liao, J. Aizpurua, Y. Luo, J. L. Yang and J. G. Hou, Nature, 2013, 498, 82-86.

28 Z. L. Zhang, P. F. Yang, H. X. Xu and H. R. Zheng, J. Appl. Phys., 2013, 113, 033102.

29 M. L. Hause, N. Herath, R. S. Zhu, M. C. Lin and A. G. Suits, Nat. Chem., 2011, 3, 932-937.

30 B. Chen, C. Yang and N. K. Goh, J. Environ. Sci., 2005, 17, 598-604.

31 Y. M. Li, J. L. Sun, H. M. Yin, K. L. Han and G. Z. He, J. Chem. Phys., 2003, 118, 6244-6249.

32 M. W. Knight, H. Sobhani, P. Nordlander and N. J. Halas, Science, 2011, 332, 702-704.

33 I. Goykhman, B. Desiatov, J. Khurgin, J. Shappir and U. Levy, Nano Lett., 2011, 11, 2219-2224.

34 A. O. Govorov, H. Zhang and Y. K. Gun'ko, J. Phys. Chem. C, 2013, 117, 16616-16631. 